\title{
Experimental Investigation of Flame Stabilization in a Turbulent Premixed Recirculation-Stabilized Jet-Flame with Simultaneous kHz Laser Diagnostics
}

\author{
Michael Severin, Oliver Lammel’ Wolfgang Meier† Manfred Aigner ${ }^{\ddagger}$ \\ German Aerospace Center (DLR) \\ Institute of Combustion Technology \\ Pfaffenwaldring 38-40, 70569 Stuttgart, Germany
}

\begin{abstract}
A turbulent premixed recirculation-stabilized jet-flame was investigated experimentally at atmospheric conditions in an optically accessible combustion chamber. The fuel was methane and the perfectly premixed stoichiometric air/methane mixture was preheated to $473 \mathrm{~K}$. The jet exit velocity was $20 \mathrm{~m} / \mathrm{s}$ corresponding to a Reynolds number of 5600 .

Simultaneous high-speed laser diagnostic measurements of stereoscopic Particle Image Velocimetry (PIV), $\mathrm{OH}^{*}$ chemiluminescence $\left(\mathrm{OH}^{*}-\mathrm{CL}\right)$ from perpendicular directions and Laser Induced Fluorescence of the $\mathrm{OH}$ radical (OH-LIF) have been performed at a repetition rate of $5 \mathrm{kHz}$ to visualize and investigate the flame stabilization mechanism qualitatively. Averaged results of the flow velocity field (PIV), the flame shape and position $\left(\mathrm{OH}^{*}-\mathrm{CL}\right)$ and the temperature distribution (OH-LIF) have been analyzed as well as time series of simultaneous single shots.

It was found that a combination of several phenomena supports flame stabilization: A consecutive flame front wrap-up at the flame root was identified as a main stabilization source. This process is preceded by spontaneous events of autoignition in the mixing layer between jet and exhaust gas prior to the flame root. Downstream of the autoignition and flame wrap-up events the flame fronts propagate into the jet flow but are constantly washed away. High strain rates due to velocity gradients have been found to inhibit reactions close to the nozzle exit. Spectral analyses showed that none of these processes occur at a dominant frequency.
\end{abstract}

\section{Nomenclature}

$\begin{array}{ll}A & \text { Surface area }\left[\mathrm{mm}^{2}\right] \\ f & \text { Frequency }[\mathrm{Hz}] \\ \boldsymbol{J} & \text { Jacobian matrix } \\ k & \text { Turbulent kinetic energy }\left[\mathrm{m}^{2} / \mathrm{s}^{2}\right] \\ \kappa & \text { Strain rate }\left[\mathrm{s}^{-1}\right] \\ \kappa_{\max } & \text { Maximum strain rate }\left[\mathrm{s}^{-1}\right] \\ \lambda & \text { Air excess ratio or eigenvalue } \\ \lambda_{\max } & \text { Largest eigenvalue } \\ \dot{m}_{\text {air }} & \text { Air mass flow }[\mathrm{g} / \mathrm{min}] \\ \dot{m}_{\mathrm{f}} & \text { Fuel }\left(\mathrm{CH}_{4}\right) \text { mass flow }[\mathrm{g} / \mathrm{min}] \\ P_{\text {th }} & \text { Thermal power }[\mathrm{kW}] \\ R e & \text { Reynolds number }\end{array}$

$\begin{array}{ll}T_{0} & \text { Preheat temperature }[\mathrm{K}] \\ t & \text { Time }[\mathrm{ms}] \\ \Delta t & \text { Time step }[\mathrm{ms}] \\ v & \text { Velocity }[\mathrm{m} / \mathrm{s}] \\ v_{\text {jet }} & \text { Nominal bulk flow jet velocity }[\mathrm{m} / \mathrm{s}] \\ x, y, z & \text { Spatial coordinates }[\mathrm{mm}] \\ \mathrm{CL} & \text { Chemiluminescence } \\ \text { LIF } & \text { Laser induced fluorescence } \\ \text { PIV } & \text { Particle image velocimetry } \\ \text { RSJF } & \text { Recirculation stabilized jet flame }\end{array}$

*Research Associate, DLR, Institute of Combustion Technology, Stuttgart, Germany, michael.severin@dlr.de. †Senior Scientist, DLR, Institute of Combustion Technology, Stuttgart, Germany.

$\ddagger$ Professor, DLR, Institute of Combustion Technology, Stuttgart, Germany. 


\section{Introduction}

Recirculation-stabilized jet-flames (RSJF) provide a promising alternative to swirl-stabilized flames in gas turbine combustion and are currently examined with regard to the application in stationary gas turbines ${ }^{1,2}$ and eventually aircraft engines. They have been shown to operate reliably with low (single digit) pollutant emissions at a wide operational range of gas turbine relevant conditions. ${ }^{3}$ Furthermore, they qualify for an operation with a large variety of both gaseous and liquid fuels. ${ }^{1,4-6}$

RSJF are used in FLOX gas turbine combustors, ${ }^{1,2,7}$ which consist of nozzles circularly arranged on the burner base plate. These nozzles feed partially premixed air and fuel into the combustion chamber at high velocity (e.g. $100 \mathrm{~m} / \mathrm{s})$. The emerging high momentum jets form a reversed flow region in the center of said circle, which feeds hot combustion products back to the jet roots. This recirculation zone is crucial for flame stabilization, since the velocities in the vicinity of the high momentum jets are typically much higher than turbulent flame speed. Furthermore, the entrainment and intense mixing of combustion products into the incoming fresh gas reduce pollutant formation by avoiding local peak temperatures.

The processes of mixing and flame stabilization in an RSJF are not fully understood yet. For example, it is still unclear whether spontaneous autoignition or the propagation of a flame front plays the dominant role in this context. In order to gain insight into these questions, a model combustor was designed, which exhibits a section of a full FLOX combustor. The model burner features a single off-centered nozzle, which is placed into a rectangular optically accessible combustion chamber. This setup was used in a preceding investigation, ${ }^{8}$ where a wide variation of operation parameters (like jet velocity or equivalence ratio) was performed and different flame stabilization regimes were identified. More information about the background and the derivation of the model burner as well as drawings and photos can be found in that preceding paper. Out of the results from the previous investigation, a single reference operation point within the RSJF regime was chosen and investigated with detailed simultaneous $\mathrm{kHz}$ laser diagnostics in the present work, in order to gain a better insight into the flame stabilization process.

\section{Experimental Setup}

A schematic drawing of the model combustor can be seen in figure 1. The combustion chamber has a cross-section of $50 \mathrm{~mm} \times 40 \mathrm{~mm}$ and a length of $600 \mathrm{~mm}$. The combustion chamber exit is an open rectangle without any exhaust gas nozzle, and the burner is operated in a lab setup at atmospheric conditions. All four sides of the combustion chamber consist of quartz glass plates of length $200 \mathrm{~mm}$, which are held together by a water cooled metal frame. The burner base plate is made of a ceramic material and the protruding jet nozzle is a tapered stainless steel pipe.

The origin for the used coordinate system is placed at the center of the burner base plate. $x$ denotes the horizontal direction, $y$ the vertical (axial) direction, and $z$ the lateral direction in which the setup is symmetric. The circular nozzle has a diameter of $10 \mathrm{~mm}$ and is off-centered by $10 \mathrm{~mm}$ in $x$ direction. The nozzle lip protrudes into the combustion chamber by $20 \mathrm{~mm}$, providing a better access for optical diagnostics at the jet root.

The complete experimental setup is shown schematically in figure 2 . The combustion air is preheated and then mixed with the fuel. For the present work, pure methane $\left(\mathrm{CH}_{4}\right)$ was used as fuel. A thermocouple monitors the temperature of the preheated gas mixture roughly $100 \mathrm{~mm}$ upstream of the nozzle exit and feedback controls the air heater.

A mounted digital single-lens reflex (DSLR) camera was used to take photos of the flame (Canon EOS 500D). For a more detailed investigation of the flame shape and position, a high speed sCMOS camera together with a high speed image intensifier (Phantom V1212 and LaVision HS-IRO) and an optical bandpass filter $(310 \pm 20 \mathrm{~nm})$ was used to record the $\mathrm{OH}^{*}$ chemiluminescence (CL) signal at a frame rate of $5 \mathrm{kHz}$ and with an intensifier gate of 20 us. ${ }^{9,10}$ The line-of-sight of this camera system was in $z$ direction, imaging the $x y$ plane with the eccentric nozzle position. Note that the $\mathrm{OH}^{*}$-CL signal

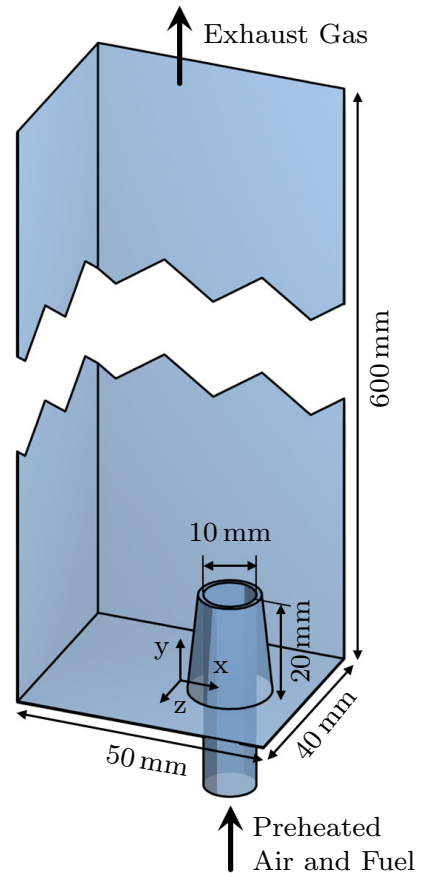

Figure 1. Model combustor with dimensions and used coordinate system. ${ }^{8}$ The nozzle axis is at $x=10 \mathrm{~mm}$ and $z=0 \mathbf{m m}$. 


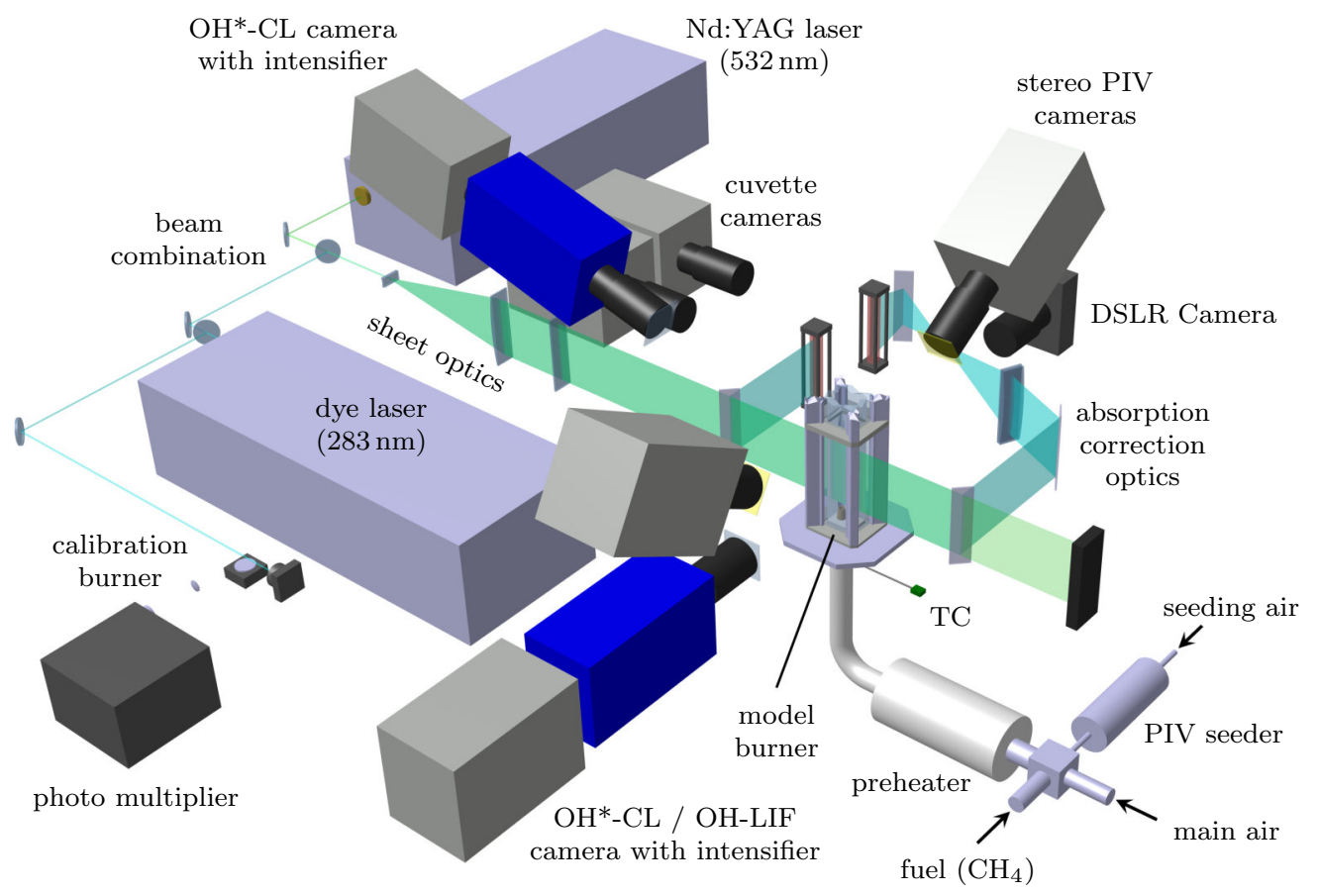

Figure 2. Schematic experimental setup. The combustion chamber is shown in a broken view for the sake of clarity.

is line-of-sight integrated, so the spatial information in $z$ is lost. To overcome this issue, a second high speed camera/intensifier/filter system was used perpendicular to the first one, imaging the $y z$ plane. This camera had to be placed at a slightly inclined angle of $12^{\circ}$ above the laser sheet.

To measure the flow velocity fields, Particle Image Velocimetry (PIV) was applied. ${ }^{11}$ For the PIV measurements, a small amount of air (about $5 \%$ of the total air flow) was fed through a fluidized bed seeder that seeded the flow with $\mathrm{TiO}_{2}$ particles. This seeding air was mixed into the main air at the same position as the fuel. The $\mathrm{TiO}_{2}$ particles have a diameter of about $1 \mu \mathrm{m}$, corresponding to a Stokes number of much less than unity, and thus follow the fluid streamlines with sufficient accuracy. ${ }^{12}$ The seeded flow inside the combustion chamber was illuminated by a high speed twin head diode-pumped Nd:YAG laser (EdgeWave InnoSlab IS200-2-LD), which was used at the same repetition rate of $5 \mathrm{kHz}$. The laser beam was formed into a sheet with a set of cylindrical lenses, resulting in a sheet of $115 \mathrm{~mm}$ height with a thickness of $1.0 \mathrm{~mm}$ in the measurement volume. The laser sheet was adjusted to match the symmetry plane of the combustor at $z=0 \mathrm{~mm}$. The double pulse separation time was $40 \mu \mathrm{s}$. Two high speed cameras (LaVision HighSpeedStar 8 ), equipped with an optical bandpass filter $(532 \pm 5 \mathrm{~nm})$, were used in double frame mode to record the PIV images. The cameras were inclined at an angle of $30^{\circ}$ in order to perform stereoscopic PIV, resulting in all three velocity components. The PIV images were processed with a commercial software (LaVision DaVis 8.3) on a single shot basis, and statistically analyzed in MatLab.

The two-dimensional Laser induced fluorescence (LIF) of the $\mathrm{OH}$ radical was measured to obtain a qualitative information on the fluid's thermochemical state (unburned/burned). ${ }^{13,14}$ Moreover, the $\mathrm{OH}$ LIF intensity can be seen as a qualitative temperature information of the burned gas. ${ }^{15}$ For the OH-LIF measurements, a highspeed dye laser (Sirah Credo Dye) tuned to a wavelength of $283.305 \mathrm{~nm}$ was used to excite the $\mathrm{Q}_{1}(7)$ transition within the $0 \rightarrow 1$ vibrational band of the $\mathrm{OH} A^{2} \Sigma^{+}-X^{2} \Pi$ band system. It was operated at the same repetition rate of $5 \mathrm{kHz}$ as the PIV and the $\mathrm{OH}^{*}$-CL setup, and the LIF laser pulse was placed in between the two PIV pulses, to get a truly simultaneous measurement. The LIF laser beam was combined with the PIV laser beam ahead of the sheet forming optics (see figure 2), so that a sheet in the same direction with a similar height is formed. This simplifies the experimental setup, but has the disadvantage that the laser sheets cannot be adjusted independently, so that the sheet optimization (parallelism, focus, ...) is always a compromise between both wavelengths. The thickness of the LIF laser sheet in the combustion chamber was approximately $0.4 \mathrm{~mm}$. The emerging OH-LIF signal was then captured 


\begin{tabular}{lccc}
\hline Air mass flow & $\dot{m}_{\text {air }}$ & {$[\mathrm{g} / \mathrm{min}]$} & 62.8 \\
Fuel $\left(\mathrm{CH}_{4}\right)$ mass flow & $\dot{m}_{\mathrm{f}}$ & {$[\mathrm{g} / \mathrm{min}]$} & 3.64 \\
Air excess ratio & $\lambda$ & & 1.0 \\
Preheat temperature & $T_{0}$ & {$[\mathrm{~K}]$} & 473 \\
Jet bulk velocity & $v_{\text {jet }}$ & {$[\mathrm{m} / \mathrm{s}]$} & 20.0 \\
Adiabatic flame temperature & $T_{\text {ad }}$ & {$[\mathrm{K}]$} & 2310 \\
Thermal power & $P_{\text {th }}$ & {$[\mathrm{kW}]$} & 3.04 \\
Reynolds number & $R e$ & & 5640 \\
\hline
\end{tabular}

Table 1. Operation conditions of the investigated flame.

by the same filter/intensifier/camera system that was used for $\mathrm{OH}^{*}$-CL (i.e. this camera recorded a double image for each cycle, similar to the PIV cameras). The only change was a reduced exposure gate time of $100 \mathrm{~ns}$ for the LIF frame. A small portion of the LIF laser sheet ahead and after the measurement volume was reflected into a dye reference cuvette and the luminescence of each cuvette was imaged by another high speed camera $(2 \times$ LaVision HighSpeedStar 5$)$, to determine the intensity distribution of the laser sheet. The intensity distribution in the first cuvette was then used to normalize the LIF signal to the incoming laser energy (in the vertical direction). The intensity distribution in the second cuvette was used to normalize the LIF signal to the laser attenuation caused by the $\mathrm{OH}$ absorption in beam direction. To distinguish between the laser attenuation by $\mathrm{OH}$ absorption and by other disturbing factors (e.g. window staining), a reference measurement was performed with the LIF laser tuned to a wavelength slightly off the $\mathrm{OH}$ resonance line. The processing and statistical analysis of the LIF images was done with an in-house MatLab code.

20000 images (or double images) were recorded for each OH*-CL, PIV and $\mathrm{OH}-\mathrm{LIF}$ in one measurement series, so the total measurement time was $4 \mathrm{~s}$. The resulting simultaneous data for each instant of time were two line-of-sight integrated $\mathrm{OH}^{*}$-CL images (from perpendicular sides), one planar three-component flow velocity field and one planar OH-LIF distribution.

The burner operation conditions for the measurement were chosen based on a preceding investigation, ${ }^{8}$ where a reference point for the RSJF-regime was chosen from a wide parameter variation covering different flame stabilization regimes. The conditions are shown in table 1 and all results presented in this work are for this set of parameters. A photo of this flame in the model combustor is shown in figure 3 .

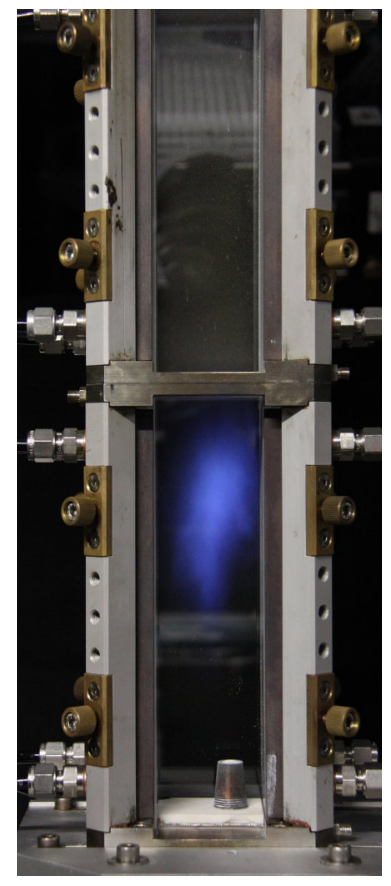

Figure 3. Photo of the investigated flame in the combustion chamber. 


\section{Results}

\section{A. Time Averaged}

From the photo in figure 3 , it is obvious that the flame is lifted and asymmetric to the nozzle axis. The flame is somewhat fluctuating but burns stably.

In figure 4 the ensemble averages over the 20000 single shots of one measurements series are shown. In subfigure a the $\mathrm{OH}^{*}$-CL average image is very similar to the flame photo, showing a lifted and asymmetric flame. The heat release zone seems widely distributed in the combustion chamber in both images. However, the $\mathrm{OH}^{*}$-CL single shots with a short exposure time show a more discrete flame front, which is highly unsteady due to turbulent fluctuations and thus seems distributed in the average image. The flame's lift-off height is approximately $60 \mathrm{~mm}$ from the nozzle exit (the flame root is at $y \approx 80 \mathrm{~mm}$ ) and the intensity maximum is at approximately $y \approx 140 \mathrm{~mm}$.

Subfigure $\mathrm{b}$ shows the corresponding averaged flow field. This result is a combination of two measurement series where the laser sheet was displaced by $-20 \mathrm{~mm}$ in $y$-direction, so that the total field of view also covers the vicinity of the nozzle exit. The high momentum jet flow exiting from the nozzle broadens and decays towards the upper end of the visible area. Flow velocities in the jet core exceed the bulk velocity of $v_{\text {jet }}=20 \mathrm{~m} / \mathrm{s}$ by more than $25 \%$. On the left side of the jet, the characteristic recirculation zone can be seen, with negative mean axial velocities up to $v_{y}=-10 \mathrm{~m} / \mathrm{s}$. This reverse flow region brings hot exhaust gas back to the root of the inflowing jet and is crucial for flame stabilization. Eliminating the flow recirculation by removing the combustion chamber lead to an immediate flame extinction. The recirculation center is at
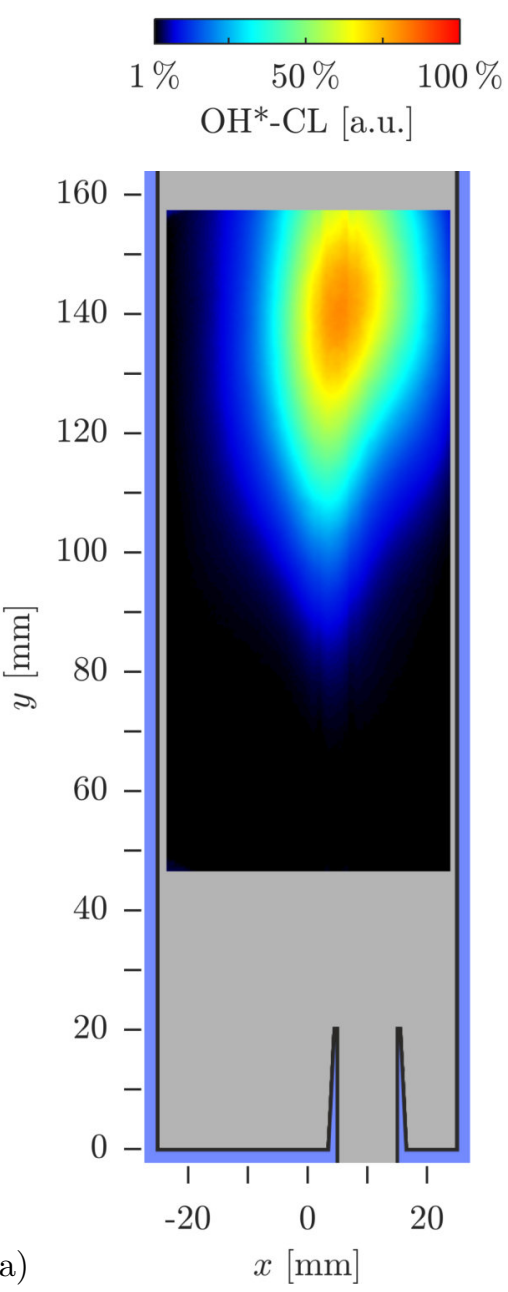
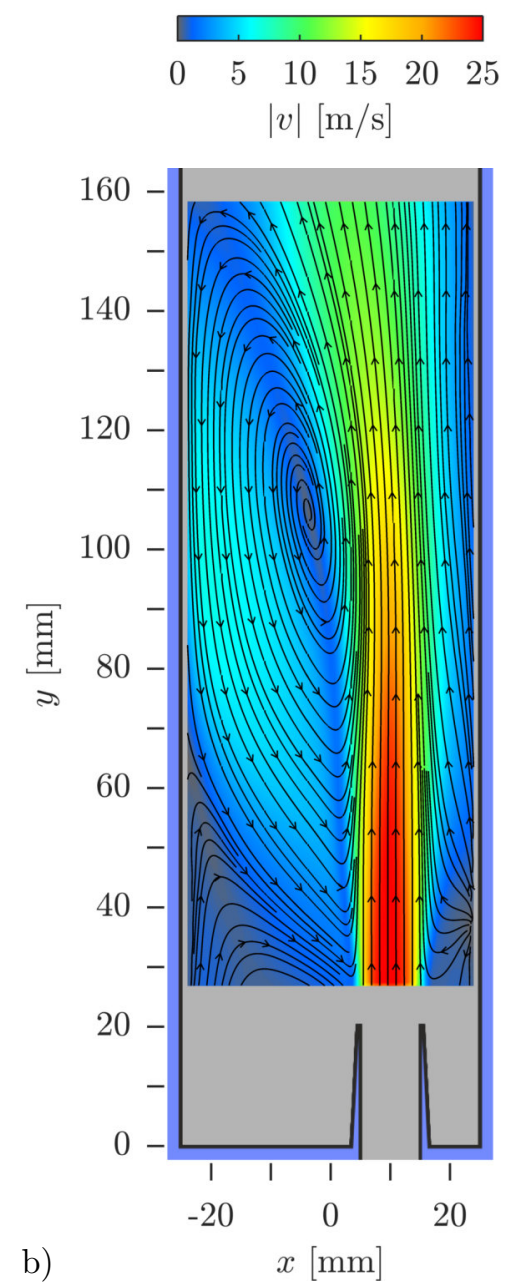
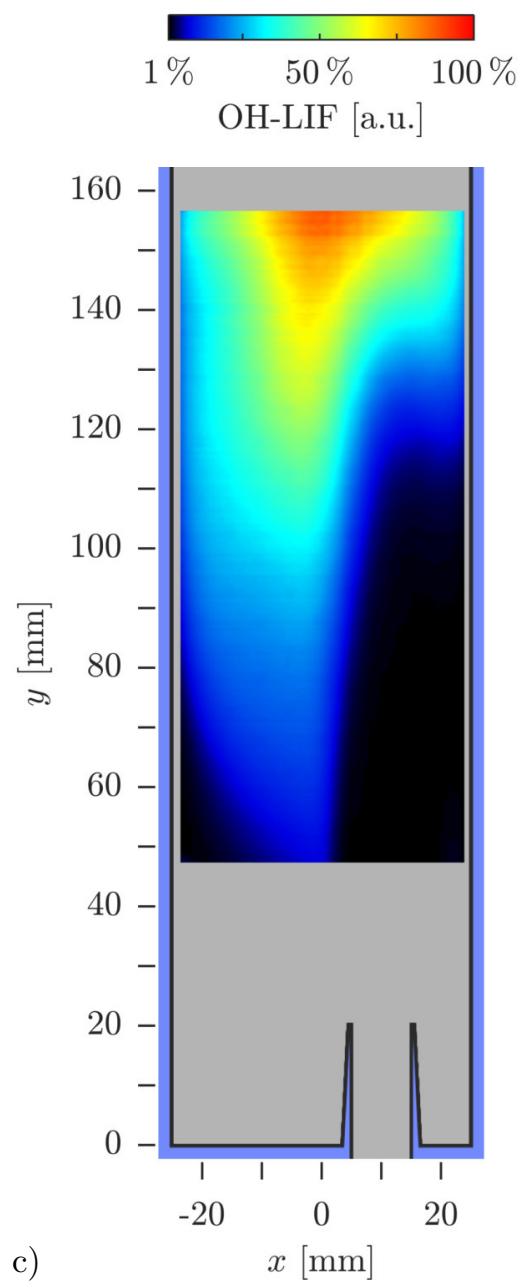

Figure 4. Averaged results of a) the flow velocity field from PIV (planar), b) the flame shape from OH*-CL measurements (line-of-sight integrated) and c) the qualitative temperature distribution from OH-LIF (planar). 
a height of $y=106 \mathrm{~mm}$. Below the large recirculation zone, a secondary recirculation bubble can be seen. From PIV-measurements in a similar burner setup, it is know that the primary recirculation encompasses the jet root outside the measurement plane, so that hot exhaust gas also reaches the opposite side of the jet. This can be seen as a source-like flow structure at around $(x, y)=(24,38) \mathrm{mm}$. Comparing subfigures a and $\mathrm{b}$ reveals, that the flame anchors at the shear layer between the jet flow and the recirculation zone, where the mean axial velocity is close to zero and where hot exhaust gas is mixed into the incoming fresh gas. The maximum of the heat release $\left(\mathrm{OH}^{*}\right.$-CL signal) however takes place further downstream in a region, where the jet has somewhat decayed, but flow velocities are still considerably high.

Subfigure 4c shows the average OH-LIF signal, which can be seen as a qualitative temperature information. From the absorption measurements it is know, that the maximum of the color scale corresponds roughly to temperatures of $2200 \mathrm{~K}$, while the minimum of the color scale corresponds to temperatures around $1500 \mathrm{~K}$. The maximum temperatures occur close to the region of the maximum heat release (in subfigure a) and the inflowing jet consists of cold unburned fluid, as expected. The high temperatures in the recirculation zone confirm the assumption, that hot exhaust gas is recirculated here. The temperature distribution also shows a significant heat loss during the recirculation. The secondary recirculation in the bottom left corner of the visible area most likely consists of exhaust gas, that has cooled down below the OH detection limit. The same holds for the surrounding fluid at the right hand side of the jet, where the OH-LIF technique cannot distinguish between cold unburned gas (in the jet) and cooled down burned gas (between the jet and the wall). Because of the low temperatures at this side, the entrainment of surrounding burned gas into the jet does not initiate reaction here and the flame becomes asymmetric to the jet axis.

It should be kept in mind that the actual physical and chemical properties of the flow field and the flame never are as smooth as they appear in the mean images. The flame is actually highly unsteady and the flow field features strong turbulent fluctuations and smaller vortices. These unsteady phenomena will be shown and investigated in the next section.

\section{B. Time-Resolved Simultaneous Single Shot Analysis}

To visualize the mixing and flame stabilization process in detail, the simultaneous OH*-CL / PIV / OH-LIF single shots are overlayed and analyzed. Figure 5 shows an example of one simultaneous single shot. On the left hand side, the $\mathrm{OH}^{*}$-CL image in the $x y$-view is shown (corresponding to figure $4 \mathrm{a}$ ). For a simple distinction between flame and non-reacting areas, a white contour line is plotted as an outline of the $\mathrm{OH}^{*}$ CL signal. In the center subfigure, the instantaneous flow velocity field is shown as colored streamlines, with the color representing the velocity magnitude (corresponding to figure $4 \mathrm{~b}$ with a changed colormap). In the second colorscale, the instantaneous OH-LIF signal is displayed (corresponding to figure 4c with a changed colormap). Here, white zones represent cold unburned gas or burned exhaust gas that has suffered a significant heat loss. Pink zones depict temperatures near the adiabatic flame temperature or superequilibrium $\mathrm{OH}$. Red, orange and yellow show burned exhaust gas with decreasing temperature due to heat losses (e.g. to the combustor wall). The interface between the fresh gas and the exhaust gas can either be interpreted as a reacting flame front or a non-reacting boundary between the two states. Although gradient methods are commonly used to identify flame fronts from OH-LIF images, the definition of the gradient threshold is usually somewhat arbitrary. Here the simultaneous OH*-CL measurement comes in handy, since $\mathrm{OH}^{*}$ exists only in the direct vicinity of the flame front. Therefore the $\mathrm{OH}^{*}$-CL contour from the left subfigure is overlayed onto the PIV/LIF data as a solid black line. Since the OH*-CL measurement is line-of-sight integrated, the contour works as an exclusive criterion: Within the contour line, reaction could take place inside the measurement plane, but the signal could as well emerge from in front of or beyond the laser plane. Outside the contour, no $\mathrm{OH}^{*}$ is detected along the entire line-of-sight, and therefore the conclusion can be drawn that no reaction happens in the laser plane. To further clarify the origin of the $\mathrm{OH}^{*}$-CL signal, the image of the second perpendicular $\mathrm{OH}^{*}-\mathrm{CL}$ measurement is shown in the right subfigure. This camera was aligned with the laser sheet and the sheet position is depicted as a dashed white line. The color scale for $\mathrm{OH}^{*}$-CL signal is the same as for the left subfigure and a white outline is displayed as well.

Taking into account the information from this third image, it becomes obvious that most of the $\mathrm{OH}^{*}$-CL signal between $y=90-120 \mathrm{~mm}$ originates from outside the measurement plane. Only at $y \approx 91 \mathrm{~mm}$ and at $y \approx 104 \mathrm{~mm}$ the laser sheet intersects the $\mathrm{OH}^{*}$-CL signal in the right subfigure. These positions correlate with the pink areas in the center subfigure and are most probably the only positions where the fresh gas / exhaust gas interface in this region is actually a reacting flame front.

Overall the two $\mathrm{OH}^{*}$-CL images in figure 5 show a much more wrinkled and disrupted flame than the 

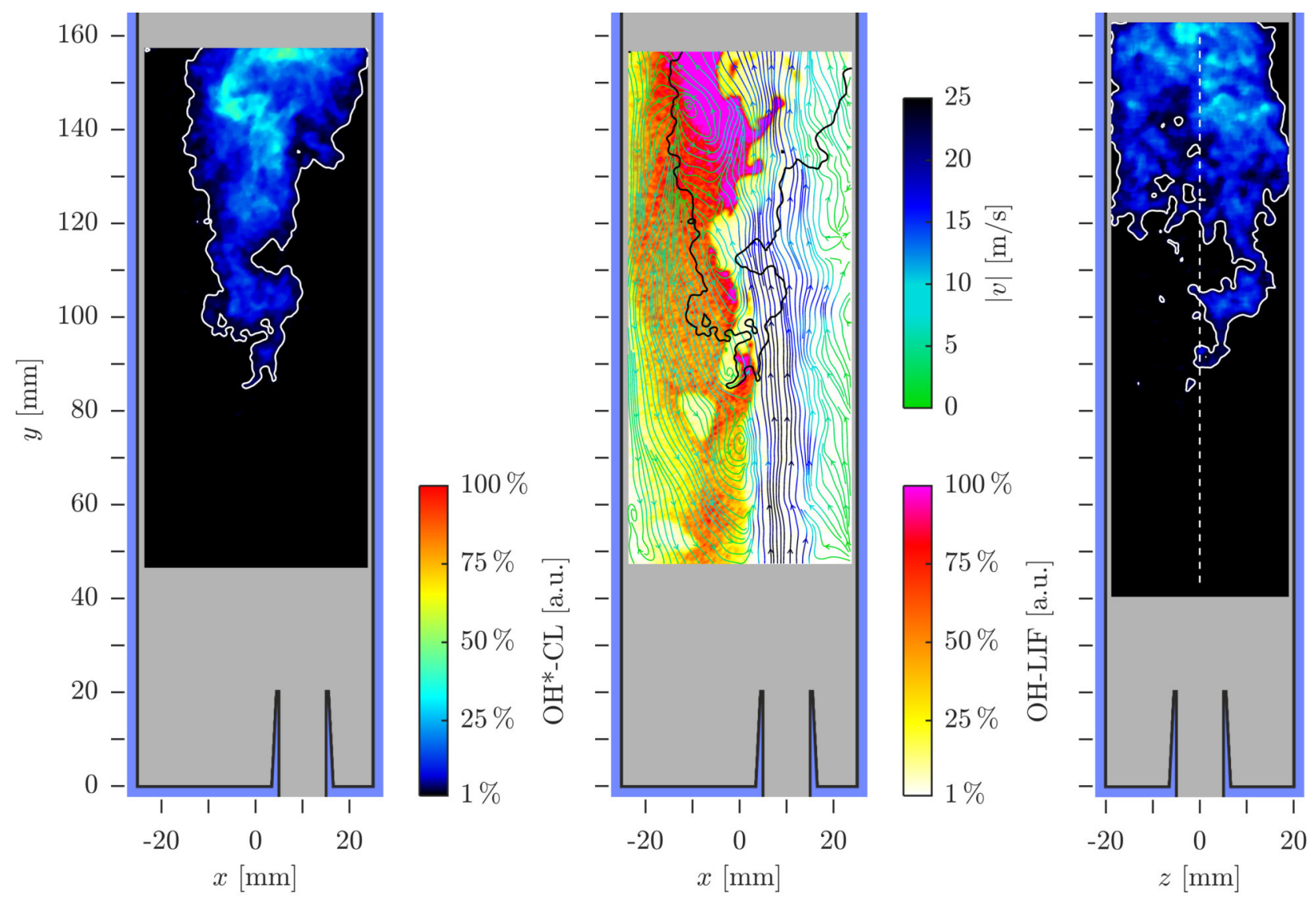

Figure 5. Example of a simultaneous single shot measurement at $t=48.6 \mathrm{~ms}$. Left: OH*-CL distribution in $x y$-view. Center: Instantaneous OH-LIF signal overlayed by color coded instantaneous streamlines from PIV in the symmetry plane $z=0 \mathrm{~mm}$. Right: $\mathrm{OH}^{*}-\mathrm{CL}$ distribution in $z y$-view with the same colorscale as the left subfigure.

average $\mathrm{OH}^{*}$-CL image in figure 4. The instantaneous flow field reveals several smaller vortices in the shear layer between the jet flow and the recirculation zone. These vortices correlate well with the interface between burned and unburned gas in the OH-LIF signal and enhance the wrap-up and mixing of these two states. The role of these vortices will further be analyzed in the following time series.

Figure 6 shows a time series of 4 simultaneous single shots. Here, the $x y \mathrm{OH}^{*}$-CL image is omitted, since its significant information is shown by the black contour line. The perpendicular $\mathrm{OH}^{*}$-CL view is shown underneath. Note that not all single shots for the full temporal resolution are shown here $(\Delta t=0.2 \mathrm{~ms})$ and the selected single shots are not equidistant in time. The series shows the characteristic shear layer vortices between the jet and the recirculation zone. The big vortex at $y \approx 105 \mathrm{~mm}$ in this series shows an example of the consecutive process of "flame wrap-up" at the flame root. The exhaust gas and the reacting flame front are wrapped around the fresh gas, which is then consumed rapidly and creates a large and intense heat release at $t=281.0 \mathrm{~ms}$. This flame wrap-up can be found in almost every single instant of time in the measurement series and seems to be mainly responsible for flame stabilization. The beginning of the next wrap-up iteration can already be seen in the image at $t=281.0 \mathrm{~ms}$ around $y \approx 100 \mathrm{~mm}$.

Between $y=80-90 \mathrm{~mm}$, an incomplete event of autoignition can be seen in the series, both in the upper and the lower row of images. Autoignition will be discussed in more detail in the next example of a time series.

In the present series in figure 6 it is furthermore interesting to track the progress of combustion downstream of the flame wrap-up: Above $y=120 \mathrm{~mm}$, reacting flame fronts can be seen even inside the high velocity jet flow. These flame fronts are constantly convected downstream, while growing and merging. This consecutive process is also visible during the entire measurement series, and is named "flame convection" for the scope of this work. Although it does not directly stabilize the flame, it seems to be responsible for 

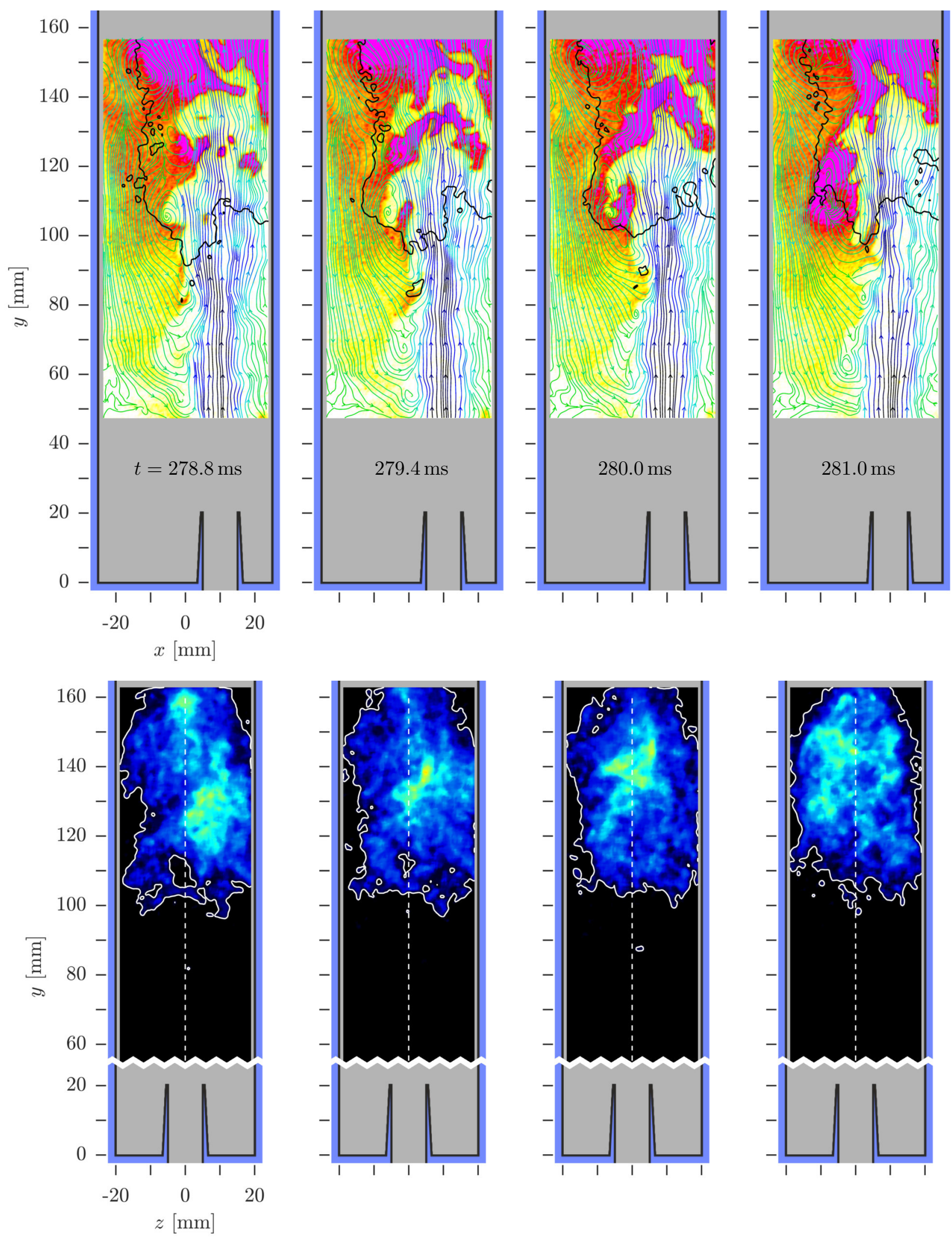

Figure 6. Time series of simultaneous single shots, showing the process of "flame wrap-up" and "flame convection". For depiction and color scales see figure 5. Top row: PIV/OH-LIF/OH*_CL in $x y$-view. Bottom row: $\mathrm{OH}^{*}$-CL in $z y$-view. 
the major part of heat release and fresh gas consumption. The hot burned gas is then partially fed into the recirculation zone (see the vortex around $(x, y) \approx(-15,150) \mathrm{mm}$ ) and thus indirectly contributes to flame stabilization.

Figure 7 shows a second time series in the same arrangement of subfigures as the previous figure. This subset was chosen, because it shows two events of autoignition, at $y=68 \mathrm{~mm}$ and at $y \approx 80 \mathrm{~mm}$ (both at $x \approx 0 \mathrm{~mm}$ and $z \approx 0 \mathrm{~mm}$ ). In the bottom $\mathrm{OH}^{*}$-CL images at $t=179.6 \mathrm{~ms}$ and $180.0 \mathrm{~ms}$ two growing ignition kernels at the position of the laser measurement plane can be seen. In the upper images, these kernels are visible in the $\mathrm{OH}^{*}$-CL-contour as well as in an increasing OH-LIF signal. Both kernels emerge at the interface between exhaust gas and fresh gas, each close to a shear layer vortex, and increase during the series. Preceding to both autoignition kernels, a strong mixing of exhaust gas and fresh gas through these vortices can be observed (at $t=179.2 \mathrm{~ms}$ ). At the end of the series at $t=180.8 \mathrm{~ms}$, the lower kernel has extinguished, while the upper kernel stabilizes and starts merging with the rest of the flame. The extinction of the lower kernel is observable by the vanishing $\mathrm{OH}^{*}$-CL signal in both orientations, although the OH-LIF signal still shows a hotspot as a remainder of the kernel (at $y \approx 60 \mathrm{~mm}$ and $t=180.8 \mathrm{~ms}$ ). Several events of autoignition can be found within the total measurement series, but they are much rarer than the consecutive events of flame wrap-up or flame convection. Therefore, it could be concluded that autoignition does not play a crucial role for flame stabilization and only supports it casually. However, the presented data does not include ignition precursors (formation of radicals other than $\mathrm{OH}$ ), and therefore no statement can be drawn about the role of incomplete autoignition, which possibly assists the flame wrap-up permanently. Furthermore, truly consecutive autoignition (without isolated events) would be almost impossible to identify with these measurement techniques, since it could not be distinguished from flame propagation.

In the upper part of the visible area, the aforementioned processes of "flame wrap-up" (at $y=100-$ $110 \mathrm{~mm}$ and $t=179.2 \mathrm{~ms}$ ) and "flame convection" can be seen again.

\section{Influence of Strain Rate}

Although several typical phenomena could be identified in the analysis of the time series, there remain some open questions: The flame wrap-up as well as autoignition events only seemed to happen at shear layer vortices at a certain height above the burner exit. Vortices closer to the nozzle exit mix fresh gas and exhaust gas, but do not initiate reaction, which in the end leads to the lifted flame. Furthermore, some autoignition kernels grow and support flame stabilization, while others extinguish. Flame speeds and ignition delay times have been calculated for the entrainment of hot exhaust gas into the jet (not shown here), but they cannot explain the lift-off height behavior of the flame.

The strain rate $\kappa$ of a flame front is defined as the change of flame surface area over time: ${ }^{16}$

$$
\kappa=\frac{1}{A} \frac{d A}{d t}
$$

For a sufficiently steady flame front this corresponds to the tangential divergence $\nabla_{t} \cdot v=\frac{\partial v_{p}}{\partial p}+\frac{\partial v_{q}}{\partial q}$ of the flow field if $p$ and $q$ are the local tangential coordinates of the flame front. To calculate the strain rate from the experimental data, it would be necessary to know the three-dimensional flame front orientation in the three-dimensional flow field. Furthermore, the strain rate could only be calculated at the actual flame position. to overcome this issue, a different approach for calculating the strain rate from the flow field was chosen here: For each vector position of the flow field, the strain tensor can be calculated as the Jacobian matrix:

$$
\boldsymbol{J}=(\nabla \boldsymbol{v})^{T}
$$

This tensor can be divided into a symmetric and an antisymmetric part. The symmetric part $\boldsymbol{S}$ contains stretch and shear, while the antisymmetric part $\boldsymbol{R}$ contains rotation.

$$
\boldsymbol{S}=\frac{1}{2}\left(\boldsymbol{J}+\boldsymbol{J}^{T}\right) \quad \boldsymbol{R}=\frac{1}{2}\left(\boldsymbol{J}-\boldsymbol{J}^{T}\right)
$$

Taking the largest eigenvalue of $\boldsymbol{S}$ provides the maximum stretch at this position.

$$
\kappa_{\max }=\lambda_{\max }(\boldsymbol{S})
$$

This basically means rotating a virtual flame front at this position into the direction of maximum strain. Similar techniques have been used in literature, for example by Shanbhogue et al. on PIV data in a swirl 

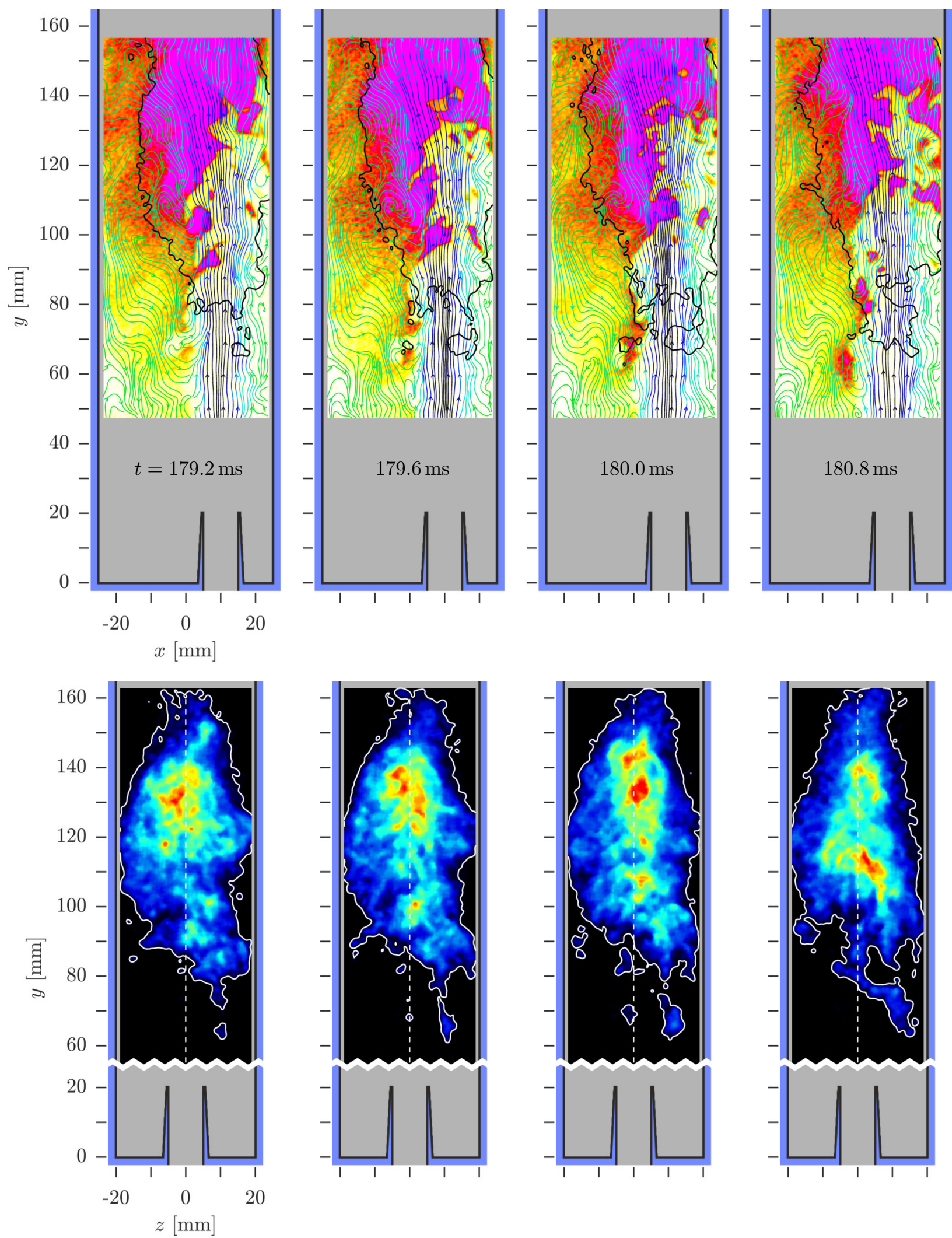

Figure 7. Time series of simultaneous single shots, showing to concurrent autoignition events. For depiction and color scales see figure 5. Top row: PIV/OH-LIF/OH*_CL in $x y$-view. Bottom row: OH*-CL in $z y$-view. 
burner. ${ }^{17}$ Detailed investigations by Sponfeldner et al. showed that a flame front indeed preferably aligns with the direction of maximum strain. ${ }^{18}$

The determined scalar $\kappa_{\max }$ can be plotted into the instantaneous flow field. An example is shown in figure 8a). The left subfigure shows an exemplary simultaneous single shot in the same representation as the previous figures. Again, the flame wrap-up can be seen (at $y \approx 110 \mathrm{~mm}$ ), as well as flame convection (above $y>120 \mathrm{~mm}$ ) and an isolated autoignition kernel (at $y=87 \mathrm{~mm}$ ). No reaction is visible at the interface of hot exhaust gas and inflowing fresh gas above and below the isolated flame kernel. In the corresponding right subfigure, the maximum stretch rates $\kappa_{\max }$ are shown. High values of $\kappa_{\max }$ follow exactly said interface up to $y<100 \mathrm{~mm}$, and are probably inhibiting flame reactions. The flame kernel at $y=87 \mathrm{~mm}$ correlates with a gap in this high $\kappa_{\max }$ contour and values here are below $\kappa_{\max }<20001 / \mathrm{s}$. The flame wrap-up and the following flame convection occur in regions of low $\kappa_{\max }$ as well.
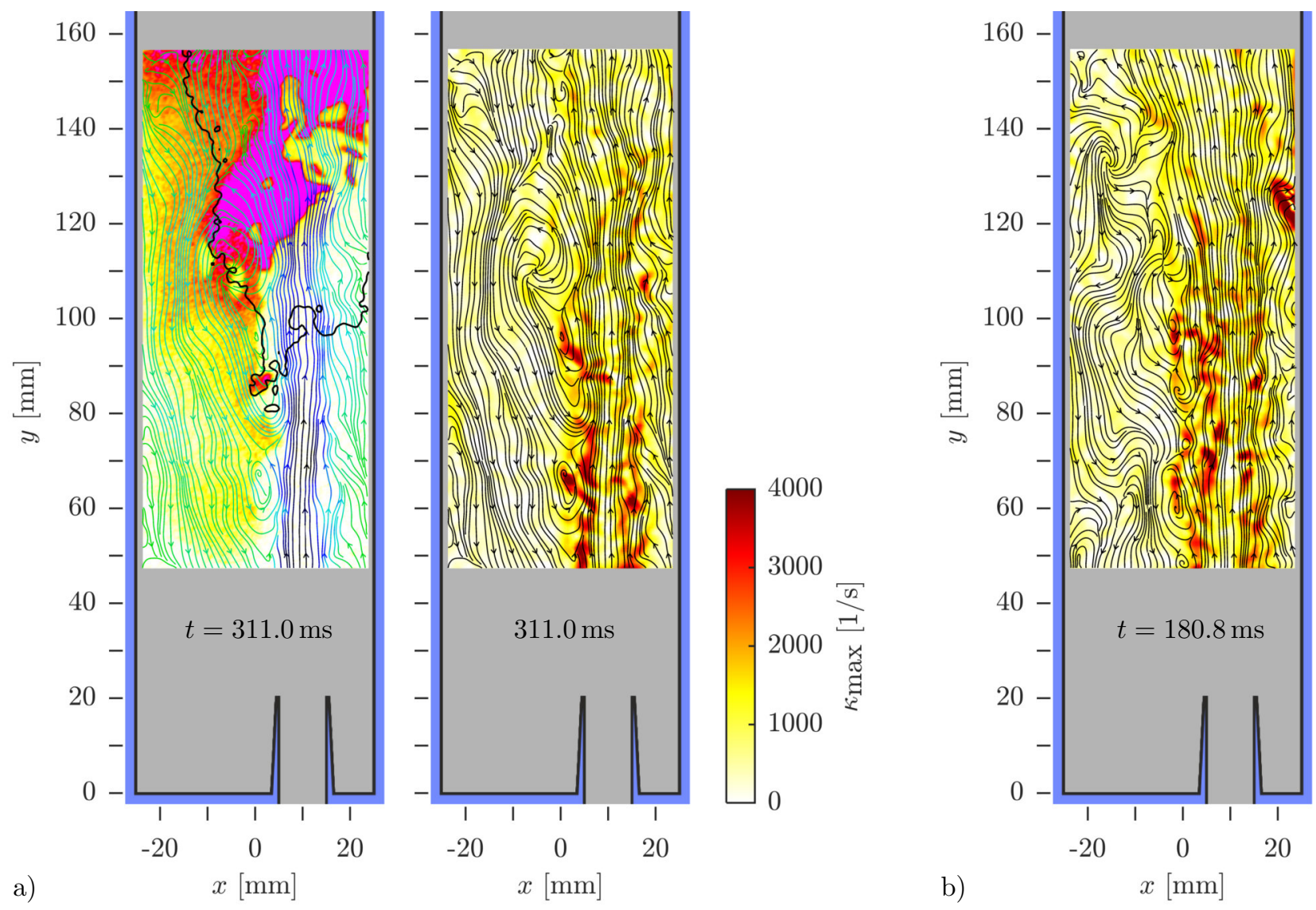

Figure 8. a) Left: Simultaneous OH-LIF/PIV/OH*_CL single shot. For depiction and scales see figure 5. Right: Color coded maximum stretch rates $\kappa_{\max }$ on top of the streamlines from the left subfigure. b) Maximum stretch rates for the last single shot of figure 7 , with the same colorscale as a).

This correlation could be found throughout the entire measurement series. It can also clarify the different behavior of the stabilizing and the extinguishing flame kernel in the previously shown series (figure 7). In figure $8 \mathrm{~b}$ ) the corresponding $\kappa_{\max }$ of the last single shot of figure 7 is displayed. The stabilizing flame kernel at $y \approx 80 \mathrm{~mm}$ corresponds to low stretch rates, while for the extinguishing kernel at $y \approx 60 \mathrm{~mm}$ the interface of burned and unburned gas is overlayed by high stretch rates. Again, the main location of flame stabilization around $y \approx 110 \mathrm{~mm}$ is a region of very low velocities and strain rates.

\section{Spectral Analysis}

The vast majority of flame wrap-up and autoignition events are linked to the observed shear layer vortices. In the instantaneous flow fields, these vortices often appear to be regularly arranged as if caused by a vortex shedding. To clarify if these vortices and the corresponding flame phenomena follow any dominant frequency, several Fourier analyses of the PIV-, OH-LIF- and OH*-CL-data have been performed. Figure 9 shows a 
spectrum of the three velocity components $v_{i}$, represented as the turbulent kinetic energy (TKE):

$$
k_{i}=\frac{1}{2} v_{i}^{\prime 2}
$$

The sum of the three components $k_{i}$ gives the total TKE $k=\frac{1}{2}\left(v_{x}^{\prime 2}+v_{y}^{\prime 2}+v_{z}^{\prime 2}\right)$. The spectrum has been evaluated at the position $(x, y, z)=(5,80,0) \mathrm{mm}$, where the velocity fluctuations in the shear layer are at most. However, neither of the velocity components in figure 9 shows any major peak of a dominant frequency. The spectrum rather is an almost ideal homogeneous turbulence. The energy dissipation towards higher frequencies follows the slope of $-\frac{5}{3}$, which is predicted by theory. ${ }^{19}$ At lower frequencies in the orders of $10^{1}$ and $10^{2} \mathrm{~Hz}$, the velocity fluctuations of the axial component $k_{y}$ are much higher than the lateral components $k_{x}$ and $k_{z}$, which are of similar magnitude. Only at higher frequencies in the order of $10^{3} \mathrm{~Hz}$, turbulence becomes truly isotropic. Since the assumption of homogeneous isotropic turbulence is the foundation of many turbulence models, this result can be valuable for numerical simulations.

The Fourier analyses of the $\mathrm{OH}^{*}$-CL and the OH-LIF signal (flame lift-off height, autoignition events, etc.) result in broadband spectra as well (not shown here). Although this lack of any dominant frequency may seem dreary from an analytical point of view, it exhibits a major advantage of RSJF-burners: No inherent instabilities are present in the flow field, and therefore one drivingpoint for detrimental thermoacoustic oscillations is

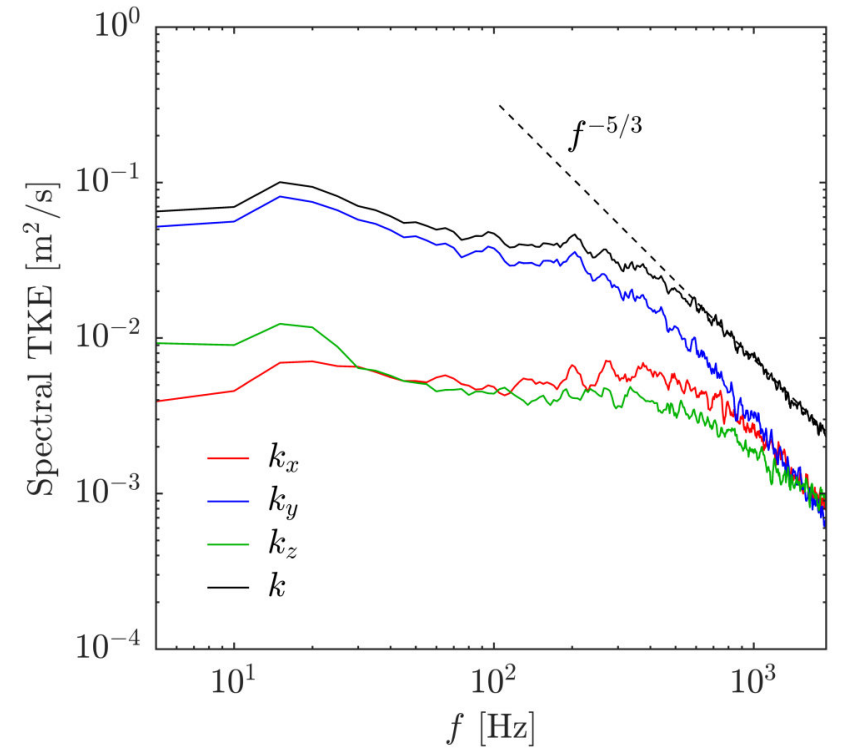
lacking.

Figure 9. Turbulence spectrum from the PIV data at position $(x, y, z)=(5,80,0) \mathbf{m m}$.

\section{Conclusions and Outlook}

Simultaneous OH*-CL / PIV / OH-LIF measurements have been performed on a premixed recirculationstabilized jet-flame to investigate flame stabilization. The flame is lifted and widely distributed. A strong internal recirculation feeds hot exhaust gas back to the flame root and supports flame stabilization. The experimental results of the present work describe this process in detail and are summarized schematically in figure 10. Smaller vortices occur in the shear layer between recirculation zone and inflowing jet. They mix hot exhaust gas into the fresh gas and increase its temperature. Sporadic autoignition events are observable at these vortices, ahead of the flame root. However, their occurrence is too rare to be mainly responsible for flame stabilization. At the flame root, a standing vortex permanently wraps up the flame front around the inflowing fresh gas. This consecutive process seems to be the main reason for stabilizing the flame root.

Parts of the flame front are drawn into the high-velocity but low-gradient jet flow, where they are convected downstream while growing and consuming fresh gas. Although the flame is permanently washed away and not self-stabilizing there, this is where the major part of fresh gas consumption and heat release takes place. In the shear layer upstream of the flame root, high velocity gradients and strain rates have been identified as a limiting factor of flame propagation or autoignition. This is probably the main reason for the flame's lift-off height. In summary, flame stabilization is a complex interaction of mixing, autoignition, "fflame wrap-up", "'flame convection"' and limitation by strain. These phenomena are not always clearly distinguishable and tend to merge. For example, if the position of an autoignition event is very close to the flame front, it becomes indistinguishable from turbulent flame propagation. This is indicated in figure 10 at the vortex below the flame wrap-up.

All these processes have been checked for periodicity, but all resulting spectra were of a broad-band turbulent nature. The lack of inherent dominant frequencies is a main advantage of an RSJF-burner, since it prevents one reason for thermoacoustic oscillations. 


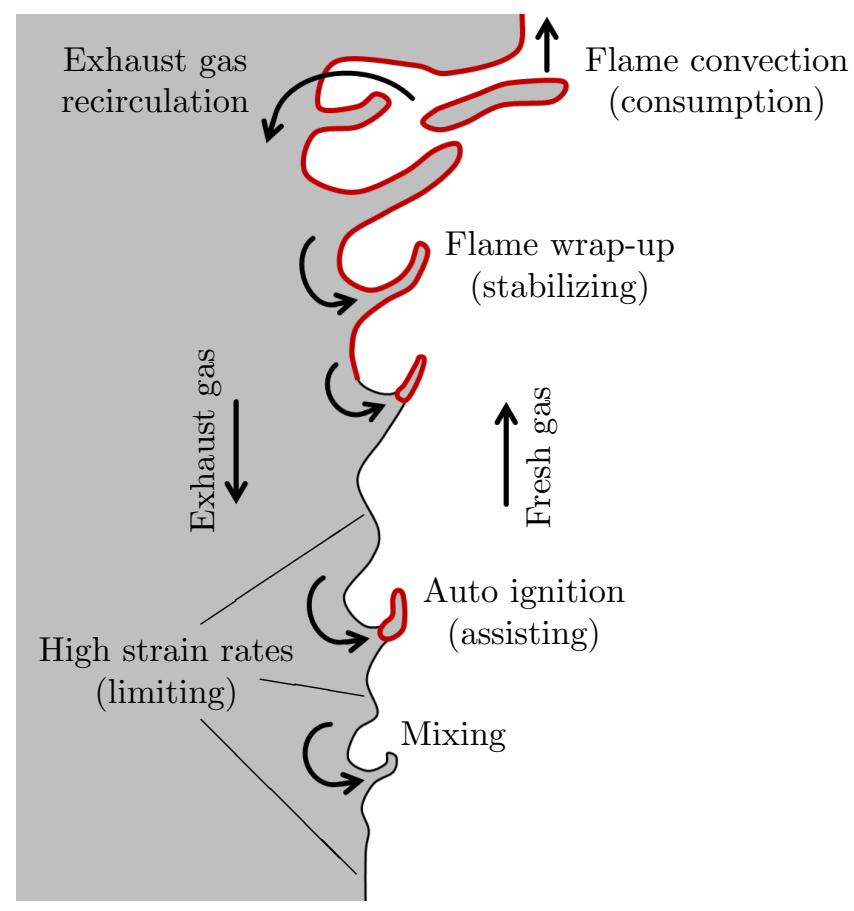

Figure 10. Summary of flame stabilization in an RSJF-burner as a sketch. The gray region depicts exhaust gas, the white region fresh gas. The black line is a non-reacting interface between fresh gas and exhaust gas, red lines indicate flame fronts.

The flame stabilization phenomena, qualitatively described in this work, could further be quantified in more detailed and focused future investigations. For example, the velocity gradients and strain rates in the shear layer close to the nozzle exit could be resolved better by PIV with a smaller field of view and be compared to known extinction strain rates or critical autoignition strain rates. Detailed numerical simulations could be helpful in further understanding the three-dimensional unsteady processes as well as the role of autoignition precursor radicals. The presented comprehensive data set can be useful as validation data for these numerical models.

\section{References}

\footnotetext{
${ }^{1}$ Lückerath, R., Meier, W., and Aigner, M., "FLOX Combustion at High Pressure with Different Fuel Compositions," Journal of Engineering for Gas Turbines and Power, Vol. 130, No. 1, 2007, pp. 011505-1 - 011505-7.

${ }^{2}$ Lammel, O., Schütz, H., Schmitz, G., Lückerath, R., Stöhr, M., Noll, B., Aigner, M., Hase, M., and Krebs, W., "FLOX Combustion at High Power Density and High Flame Temperatures," Journal of Engineering for Gas Turbines and Power, Vol. 132, No. 12, 2010, pp. 121503ff.

${ }^{3}$ Rödiger, T., Lammel, O., Aigner, M., Beck, C., and Krebs, W., "Part-Load Operation of a Piloted FLOX Combustion System," Journal of Engineering for Gas Turbines and Power, Vol. 135, No. 3, 2013, pp. 031503-1.

${ }^{4}$ Danon, B., de Jong, W., and Roekaerts, D., "Experimental and Numerical Investigation of a FLOX Combustor Firing Low Calorific Value Gases," Combustion Science and Technology, Vol. 182, No. 9, 2010, pp. 1261-1278.

${ }^{5}$ Gounder, J. D., Zizin, A., Lammel, O., Rachner, M., Kulkarni, S. R., and Aigner, M., "Experimental and numerical investigation of spray characteristics in a new FLOX based combustor for liquid fuels for Micro Gas Turbine Range Extender (MGT-REX)," 52nd AIAA/SAE/ASEE Joint Propulsion Conference, AIAA Propulsion and Energy Forum, Vol. AIAA 2016$4698,2016$.

${ }^{6}$ Lammel, O., Severin, M., Ax, H., Lückerath, R., Tomasello, A., Emmi, Y., Noll, B., Aigner, M., and Panek, L., "High Momentum Jet Flames at Elevated Pressure, A: Experimental and Numerical Investigation for Different Fuels," ASME Paper no. GT2017-64615, 2017.

${ }^{7}$ WS Wärmeprozesstechnik GmbH, "Registered trademark FLOX," 1991, German registry number 1177475.

${ }^{8}$ Severin, M., Lammel, O., Meier, W., and Aigner, M., "Flame Stabilization Regimes of Lean Premixed Confined Jet Flames at Different Reynolds Numbers," 53rd AIAA/SAE/ASEE Joint Propulsion Conference, Vol. AIAA 2017-4684, 2017.

${ }^{9}$ Dandy, D. S. and Vosen, S. R., "Numerical and Experimental Studies of Hydroxyl Radical Chemoluminescence in Methane-Air Flames," Combustion Science and Technology, Vol. 82, 1992, pp. 131 - 150.

${ }^{10}$ Najm, H. N., Paul, P. H., Mueller, C. J., and Wyckoff, P. S., "On the Adequacy of Certain Experimental Observables as Measurements of Flame Burning Rate," Combustion and Flame, Vol. 113, No. 3, 1998, pp. 312-332.
} 
${ }^{11}$ Adrian, R. J. and Westerweel, J., Particle Image Velocimetry, Cambridge University Press, 2011.

${ }_{12}^{12}$ Melling, A., "Tracer particles and seeding for particle image velocimetry," Meas. Sci. Technol., Vol. 8, 1997, pp. 14061416.

${ }^{13}$ Smyth, K. C. and Crosley, D. R., Applied Combustion Diagnostics - Detection of minor species with laser techniques, chap. 2, Taylor \& Francis New York, 2002, pp. 9-68.

${ }^{14}$ Eckbreth, A. C., Laser Diagnostics for Combustion Temperature and Species, 2nd Edition, CRC Press, 1996.

${ }^{15}$ Heinze, J., Meier, U., Behrendt, T., Willert, C., Geigle, K.-P., Lammel, O., and Lückerath, R., "PLIF Thermometry Based on Measurements of Absolute Concentrations of the $\mathrm{OH}$ Radical," International journal of research in physical chemistry and chemical physics, Vol. 225, No. 11-12, 2011, pp. 1315 - 1341.

${ }^{16}$ Law, C. K., Combustion Physics, Cambridge University Press, 2006.

${ }^{17}$ Shanbhogue, S., Sanusi, Y., Taamallah, S., Habib, M., Mokheimer, E., and Ghoniem, A., "Flame macrostructures, combustion instability and extinction strain scaling in swirl-stabilized premixed $\mathrm{CH}_{4} / \mathrm{H}_{2}$ combustion," Combustion and Flame, Vol. 163, 2016.

${ }^{18}$ Sponfeldner, T., Boxx, I., Beyrau, F., Hardalupas, Y., Meier, W., and Taylor, A., "On the alignment of fluid-dynamic principal strain-rates with the 3D flamelet-normal in a premixed turbulent V-flame," Proceedings of the Combustion Institute, Vol. 35, No. 2, 2015, pp. 1269-1276.

${ }^{19}$ Pope, S. B., Turbulent Flows, Cambridge University Press, 2000. 